\title{
Evaluation of ECLIA antigen detection tests as screening methods for COVID-19 in comparison with molecular analysis
}

\author{
Olga Kolesova ${ }^{1,3} \cdot$ Flaminia Tomassetti $^{2,3} \cdot$ Paola Cerini $^{3} \cdot$ Davide Finucci $^{3} \cdot$ Giordano Turchetti $^{3}$. \\ Francesca Capogreco ${ }^{3}$. Sergio Bernardini ${ }^{4,5} \cdot$ Graziella Calugi $^{3} \cdot$ Massimo Pieri $^{3,4,5}$
}

Received: 28 September 2021 / Accepted: 16 November 2021 / Published online: 30 November 2021

(c) The Author(s), under exclusive licence to Royal Academy of Medicine in Ireland 2021

\begin{abstract}
Background Data from literature shows that antigen tests are rapid and helpful tools for diagnosis of COVID-19.

Aim This work aimed to evaluate the performances of the Elecsys SARS-CoV-2 Antigen test, in comparison to RT-qPCR, the gold standard.

Methods A total of 110 swabs were tested; according to rRT-PCR, 76 were positive, and 34 were negative. The swabs were processed by Elecsys SARS CoV 2 Antigen assay (Roche Diagnostics GmbH, Mannheim, Germany), an electrochemiluminescence immunoassay (ECLIA).

Results In a first evaluation, the overall sensitivity and specificity were $85 \%$ and $100 \%$, respectively. It was noted that most of the discordant cases had cycle threshold $(\mathrm{Ct})$ values $>28$. Therefore, it was assumed a new measure to evaluate sensitivity and specificity, then samples with $\mathrm{Ct}$ values $<28$ were selected. In this way, it was achieved a $\mathrm{Ct}<28$ sensitivity of $94 \%$. The level of agreement between the two tests was $89.1 \%$ with $\kappa$ value of 0.77 for total data and $95.9 \%$ with $\kappa$ value of 0.95 for samples with $<28 \mathrm{Ct}$. The antigen test performs well in the presence of high viral loads, whereas lower levels are missed. Conclusions The comparison data obtained in this study support that this method seems a proper approach for rapid screening of patients with high SARS-CoV-2 viral load; however, the rate of sensitivity is highly Ct-dependent.
\end{abstract}

Keywords Antigen test $\cdot$ COVID-19 $\cdot$ Diagnostic methodology $\cdot$ Molecular analysis $\cdot$ SARS-CoV-2

\section{Introduction}

In the ongoing pandemic context of COVID-19, diagnostic testing for SARS-CoV-2 is crucial to limit the spread of the virus as well as appropriately manage infected patients [1].

Olga Kolesova1 and Flaminia Tomassetti equally contributed to the work.

Massimo Pieri

massimo.pieri@uniroma2.it

1 Department of Public Health and Infectious Diseases, University of Rome, La Sapienza, Rome, Italy

2 Department of Life Sciences and Biotechnology, University of Ferrara, Ferrara, Italy

3 Lifebrain Srl, Guidonia Montecelio, Viale Roma 190/A, Rome, Italy

4 Department of Experimental Medicine, University of Tor Vergata, Via Montpellier 1, 00133, Rome, Italy

5 Clinical Biochemistry, Tor Vergata University Hospital, Rome, Italy
Different diagnostic test manufacturers have developed rapid tests based on SARS-CoV-2 proteins detection in respiratory samples [2]. Quantitative reverse transcription-polymerase chain reaction (RT-qPCR) is the main diagnostic method to identify patients with COVID-19; however, this method is time-consuming and required a properly trained laboratory staff. For these reasons, the demand for a fast and easy-touse diagnostic method to shorten the time of result-reporting is even more needed [3].

Viral antigens are only expressed when the virus is actively replicating, making antigen tests clinically useful for identifying acute or early infection [4]. Current research [5-7] suggests that highly sensitive immunological diagnostic methods directly detect viral antigens in clinical samples which would be very helpful for rapid and accurate diagnosis of COVID-19. However, these antigen tests' analytical performances depend on different factors, including the viral load, the quality of the specimen, and how it is processed. The performance also depends on the setting of patients tested [8]. 
This evaluation aimed to assess the antigen diagnostic test performances as a timely and effortless detection method, compared with molecular technique. We investigated Elecsys SARS-CoV-2 Antigen Test (Roche Diagnostics GmbH, Mannheim, Germany), an electrochemiluminescence immunoassay (ECLIA), through sensitivity and specificity, compared by molecular positive results, obtained by the gold standard method, the rRT-PCR. The Elecsys SARS CoV 2 Antigen assay is an immunoassay for the in vitro qualitative detection of the nucleocapsid $(\mathrm{N})$ antigen of SARS-CoV-2 in nasopharyngeal and oropharyngeal swab samples.

\section{Materials and methods}

\section{Clinical specimens}

Nasopharyngeal swab specimens were collected from samples of Lifebrain laboratory, coming from paucisymptomatic and asymptomatic patients. A total of 110 patients were enrolled in collection of samples, including 52 females and 58 males with a median age of 48 years old (range 10-87). The study has been led in accordance with the Declaration of Helsinki, under the terms of relevant local legislation.

\section{Molecular analysis}

A total of 110 nasopharyngeal swabs samples were obtained at the different Lifebrain laboratories sites from either symptomatic patients or patients with high-risk exposure to COVID-19. The swabs were collected in $2 \mathrm{ml}$ of viral preservation medium in Kang Jian Virus Collection and Preservation System (Jiangsu Kangjian Medical Apparatus Co., Ltd., China). All samples were tested within $24 \mathrm{~h}$ of collection. Viral RNA was extracted starting from $200 \mu \mathrm{l}$ of swab fluid using the MGISP-960 High-throughput Automated Sample Preparation System using MGIEasy Magnetic Beads Virus DNA/RNA Extraction Kit. The detection of SARS-CoV-2 RNA was carried out using the MutaPLEX ${ }^{\circledR}$ Coronavirus (SARS-CoV-2) real-time RT-PCR Kit for the simultaneous in vitro detection of RNA of novel coronavirus (SARS-CoV-2) and other betacoronaviruses, extracted from biological specimens. The test also has a systemic control based on the human $\beta$-actin gene: only by (separate) amplification of this gene, the correct and sufficient sample collection from the patient is proven. MutaPLEX ${ }^{\circledR}$ Coronavirus (SARS-COV-2) kit (Immundiagnostik AG, Germany) detects three viral genes: SARS-CoV-2 specific-S and RdRP genes, and $\mathrm{E}$ gene that is characteristic for both known SARS viruses. The cycling conditions were as follows: $10 \mathrm{~min}$ at $45{ }^{\circ} \mathrm{C}, 5 \mathrm{~min}$ at $95{ }^{\circ} \mathrm{C}, 45$ cycles $\times[10 \mathrm{~s}$ at $95^{\circ} \mathrm{C}, 40 \mathrm{~s}$ at $\left.60^{\circ} \mathrm{C}\right]$. Samples positive for SARS-CoV-2 are characterized by a cycle threshold $(\mathrm{Ct})$ value at or below 34 cycles of RdRp gene.

\section{Antigen test}

The present study evaluated an electrochemiluminescence immunoassay (ECLIA) antigen test, Elecsys SARS CoV 2 Antigen assay. To determine if SARS-CoV-2 antigen could be detected in the same PCR-specimens, a 1:1 dilution was applied for antigen samples. Briefly, $200 \mu \mathrm{l}$ from ready inactivated swab form Preservation System (Jiangsu Kangjian Medical Apparatus Co., Ltd., China) were pipetted and mixed to $200 \mu \mathrm{l}$ of SARS CoV-2 Roche Extraction Solution to SARS-CoV-2 antigen test. The Elecsys SARS-CoV-2 Antigen assay uses monoclonal antibodies directed against the SARS-CoV-2 N protein in a double-antibody sandwich assay format to detect SARS-CoV-2 in upper respiratory tract specimens. The specificity and sensitivity declared by the manufacturer was respectively $99.9 \%$ and $94.5 \%$. This assay is intended for use on Cobas $\mathrm{E}$ immunoassay analysers. In this study, Cobas E 602 analyzer has been used as analytic platform.

\section{Statistics}

The sensitivity and specificity of antigen test were evaluated, assuming as gold standard the rRT-PCR. Analyses were performed using MedCalc 19.2.0 (MedCalc Software Ltd, Ostend, Belgium). The level of agreement between the two assays was evaluated by Cohen's kappa coefficient $(\kappa)$, assuming a substantial agreement with a $\kappa$ value of 0.01-0.20 slight agreement; $0.21-0.40$ fair agreement; $0.41-0.60$ moderate agreement; $0.61-0.80$ substantial agreement; and 0.81-1.00 almost perfect or perfect agreement.

\section{Results}

It was assumed RT-PCR results as true positive (TP) and true negative (TN) for evaluation of sensitivity and specificity. According to RT-qPCR results, 34 samples were negative, and 76 were positive, with a range of $\mathrm{Ct}$ value from 14 to 40 . After the molecular analysis, the samples were evaluated by the antigen assay.

Figure 1 shows all negatives and all positives detected by the antigen test. In Fig. 1A, an $85 \%$ for concordant molecular with positive antigen samples was observed, instead of molecular and negative antigen samples, which have shown a level agreement of $100 \%$. Figure $1 \mathrm{~B}$ shows $\mathrm{Ct}<28$ positive values, which have a $94 \%$ value for concordant molecular with positive antigen samples, higher than the first ones. The negative results did not change their level of agreement. 


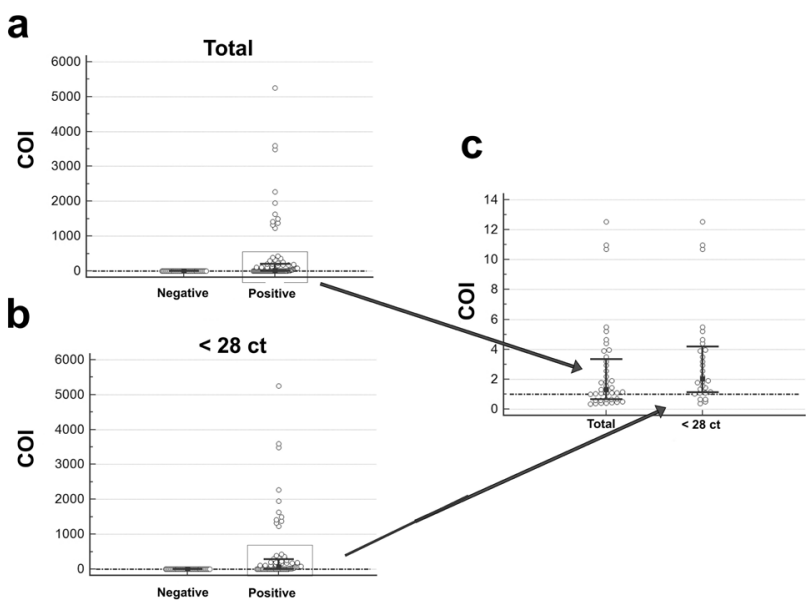

Fig. 1 a Graphical representation of total positive and negative antigen results according to RT-PCR Ct values. b Graphical representation of $\mathrm{Ct}<28$ positive and negative antigen results according to RTPCR Ct values. $\mathbf{c}$ is an enlargement section of positive; it is possible to see the significant number of false positive, detected by antigen method, and the decrease of antigen false positive numbers in confront of the total samples, under the cut-off line $(\mathrm{COI}=1)$

As can be seen from the enlargement (Fig. 1C), assuming a Ct value $<28$, the test general sensitivity can be increased.

Among the 76 positive samples, the Elecsys SARSCoV-2 Antigen Rapid Test detected 64 samples as a positive sample, 12 as negative sample. The overall sensitivity, evaluated with ROC curve, was $85 \%$ (Fig. 2A). The 34 samples with a negative result with RT-qPCR technique were also negative with the rapid test, giving an overall specificity of $100 \%$. The cut-off considered was $>1$ and the area under the curve (AUC) was 0.943 , with $p<0.001$.

The 64 concordant cases had a $\mathrm{Ct}$ values range between 14 and 28, whereas the 12 discordant cases had a Ct values a
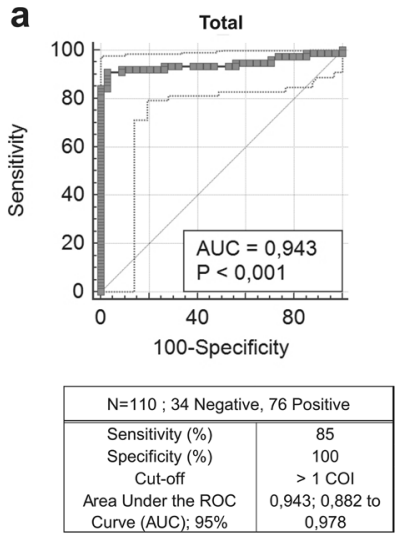

b
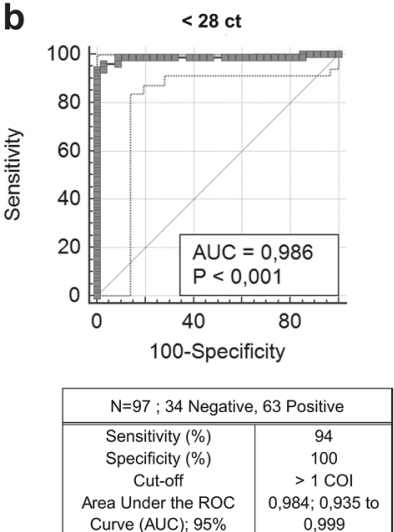

Fig. 2 a Sensitivity and specificity of total samples $(n=110)$ based of ROC curve. The AUC is 0.943 with $p<0.001$. b Sensitivity and specificity of $\mathrm{Ct}<28$ samples $(n=97)$ based of ROC curve. The AUC is 0.986 with $p<0.001$ ranging between 28 and 37 . Based on this observation, it was decided to consider $\mathrm{Ct}$ values $<28$ samples.

According to these new criteria, Elecsys SARS-CoV-2 Antigen test achieved a sensitivity of $94 \%$, while the specificity persists at $100 \%$ (Fig. 2B). The cut-off considered was $>1$ and the area under the curve (AUC) was 0.986, with $p<0.001$. Both overall and $\mathrm{Ct}$ values $<28$ specificity and sensitivity were evaluated with ROC curve with $95 \%$ confidence interval (CI). The level of agreement between the two assays was evaluated by Cohen's kappa coefficient $(\kappa)$, assuming a substantial agreement with a $\kappa$ value of 0.77 and a percentual of agreement of $89.1 \%$ for total data and a perfect agreement with $\kappa$ value of 0.92 and percentual of agreement: $95.9 \%$ for samples with $<28 \mathrm{Ct}$. In fact, in Fig. 3, we can observe a graphical representation of positive and negative antigen results according to $\mathrm{Ct}$ values. The trend of antigen load is Ct-dependent and highlights that a significant difference $(p<0.001)$ in the $\mathrm{Ct}$ value is noted between the positive and negative tests for antigen method: the concordance is spread in a range between 18 and $28 \mathrm{Ct}$ values. Therefore, only samples with $\mathrm{Ct}<28$ were considered in order to increase sensitivity.

\section{Discussion}

SARS-CoV-2-specific antigen assays, a fast and straightforward alternative for nucleic acid amplification assays, have become available recently. Here, we describe the comparison of molecular swabs and Elecsys SARS-CoV-2 Antigen test.

As already known from other studies $[6,9,10]$, antigenic tests' performance is highly variable, and it is mostly $\mathrm{Ct}$ values-related. If samples with a vast range of $\mathrm{Ct}$ values are

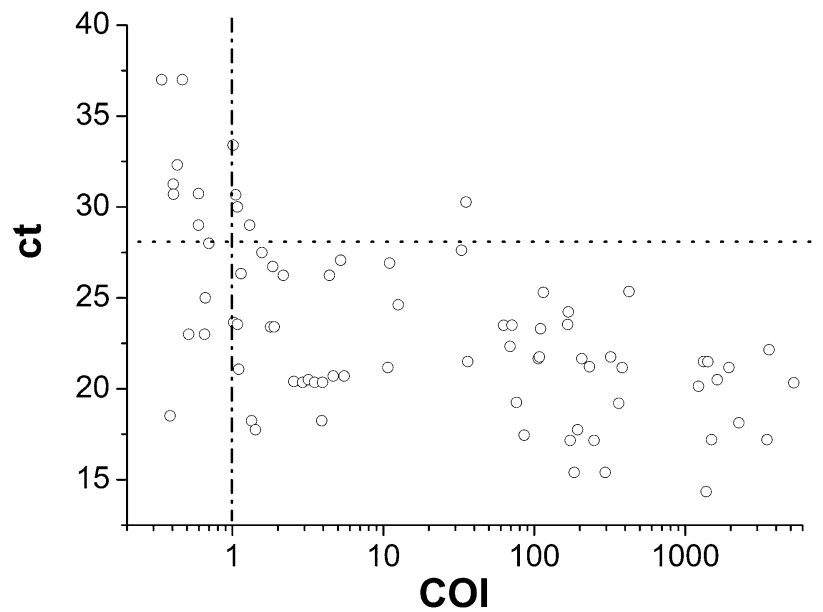

Fig. 3 Graphical trend of the amount of antigen (in COI) obtained on the antigen assay based on $\mathrm{Ct}$ values. The dashed line represents the cut-off of the antigen test $(\mathrm{COI}=1)$. The dotted line shows the $\mathrm{Ct}$ value adopted, set to $\mathrm{Ct}$ value $<28$ 
analyzed, the antigen test's sensitivity drops to minimum percentages $[8,11]$. Instead, if samples with a specific range of $\mathrm{Ct}$ values (usually $\mathrm{Ct}<25$ ) are analyzed, the diagnostic capacity of antigen test will increase [12] or will correspond to specificity and sensitivity obtained with PCR [13].

The results also showed low sensitivity for a wide range of $\mathrm{Ct}$ values but excellent specificity. For this, according to the literature, we had thought to discern samples according to their $\mathrm{Ct}$ value and we chose all the samples with $\mathrm{Ct}$ value $<28$. The sensitivity and the specificity evaluated with this range achieved significant percentages.

However, the data obtained deviated from the indications of manufacturer. The sensitivity valued with a wide range of $\mathrm{Ct}$ value and $\mathrm{Ct}$ value $<28$ is always lower than sensitivity declared by manufacturer. We supposed that this discrepancy is due to the limited number of samples. Moreover, samples from this study have been collected in a private structure, Lifebrain laboratories network, where most patients were paucisymptomatic or asymptomatics, with low viral load. The low viral load could have influenced the performance of the antigen method, decreasing sensitivity. Anyhow, the $\mathrm{Ct}$ value $<28$ results have been demonstrated good sensitivity and specificity, respectively, $94 \%$ and $100 \%$. The cases over $\mathrm{Ct}<28$ line were detected as false positives by antigen test most likely due to a low level viremia. Although, cases under $\mathrm{Ct}<28$ line were correctly detected as positive cases. The $\mathrm{Ct}<28$ line divided the samples in samples that have virus in a replicating phase, where virus overexpresses $\mathrm{N}$ protein, easily detected by antigen test and, in samples that have mostly very high $\mathrm{Ct}$ values, suggesting that virus is in a non-replicative phase and $\mathrm{N}$ protein is low or not at all expressed. This hypothesis could explain the discrepancies; however, it is not the only reason, since antigen test diagnostic performance is strongly influenced by other variables, as clinical features, and sampling time.

Furthermore, we tried to evaluate the antigen test both on single samples and on the same molecular swab samples. The values of sensitivity and specificity were equivalent for both samples. This feature is an advantage of the Roche antigen test because it needs the same molecular analysis sample. For these criteria, a single collection is sufficient; this benefit meets the patient's satisfaction and needs. The patients do not have to come back to the laboratory for a confirmatory test swab. The already presence of swab in the laboratory allows to accelerate the turnaround time (TAT), decrease the laboratory's costs, and simplify laboratory workflow.

Overall, the data of comparison between Elecsys SARSCoV-2 Antigen test support that this method seems a proper approach for patients with high SARS-CoV-2 viral load. The antigen test gives the best results in cases with high viral load, in pre-symptomatic and early symptomatic cases up to 5 days from the onset of symptoms. Moreover, antigen test plays an important role in population screening, especially because they are not affected by new variants on spike (S) protein of SARS-CoV-2. Therefore, their target is $\mathrm{N}$ protein during virus replicating phase, and then they are not subjected to new variants, being not influenced by $\mathrm{S}$ protein mutation due to virus adaptation [14]. Despite molecular analysis is still the gold standard for diagnosing SARS-CoV-2 infection, antigen test proves to be valuable tools for screening investigations, especially in closed community with high prevalence of spread, symptomatic, and asymptomatic healthcare workers with significant exposure, confirmed case contacts, and symptomatic people [15].

\section{Conclusion}

In conclusion, Roche Elecsys SARS-CoV-2 Antigen test has shown excellent performance to assist in the SARS-CoV-2 screening and diagnosing, achieving good percentages of specificity and sensitivity. However, it depends on multiple factors as viral loads, clinical status, and symptomaticity, which could significantly influence the final performance of antigen test. Nevertheless, due to shorter response times, low costs, and good screening performance, antigen test is a useful diagnostic tool during the COVID-19 pandemic.

Whereas molecular analysis is the gold standard, Roche Elecsys SARS-CoV-2 Antigen test represents an excellent diagnostic solution. The possibility of double test on the same swab for confirmatory analysis increases the overall lab workflow.

Author contribution Conceptualization: Graziella Calugi, Sergio Bernardini, Massimo Pieri; formal analysis and investigation: Olga, Kolesova, Paola Cerini, Davide Finucci, Giordano Turchetti; writingoriginal draft preparation: Olga Kolesova, Flaminia Tomassetti, Massimo Pieri; writing — review and editing: Sergio Bernardini, Graziella Calugi, Massimo Pieri, Flaminia Tomassetti; supervision: Massimo Pieri, Graziella Calugi, Francesca Capogreco, Sergio Bernardini.

Funding Not applicable.

Availability of data and material All the data and materials were described in the "Materials and methods" section.

Code availability Not applicable.

\section{Declarations}

Conflict of interest The authors declare no competing interests.

\section{References}

1. Li L, Tan C, Zeng J et al (2021) Analysis of viral load in different specimen types and serum antibody levels of COVID19 patients. J Transl Med 19(1):30. https://doi.org/10.1186/ s12967-020-02693-2 
2. Center for Disease Control and Prevention (2020) https://www. cdc.gov/coronavirus/2019-ncov/lab/resources/antigen-testsguidelines.html

3. Cerutti F, Burdino E, Milia MG et al (2020) Urgent need of rapid tests for SARS CoV-2 antigen detection: evaluation of the SD-Biosensor antigen test for SARS-CoV-2. J Clin Virol 132:104654. S1386-6532(20)30396-6. https://doi.org/10.1016/j. jcv.2020.104654

4. WHO (2020) https://www.who.int/publications-detail-redirect/ diagnostic-testing-for-sars-cov-2

5. Chaimayo C, Kaewnaphan B, Tanlieng N et al (2020) Rapid SARS-CoV-2 antigen detection assay in comparison with real-time RT-PCR assay for laboratory diagnosis of COVID19 in Thailand. Virol J 17(1):177. https://doi.org/10.1186/ s12985-020-01452-5

6. Kruttgen A, Cornelissen CG, Dreher M et al (2021) Comparison of the SARS-CoV-2 rapid antigen test to the real star Sars-CoV-2 RT PCR kit. J Virol Methods 288:114024. S01660934(20)30276-7 [pii]. https://doi.org/10.1016/j.jviromet.2020. 114024

7. Mak GC, Cheng PK, Lau SS et al (2020) Evaluation of rapid antigen test for detection of SARS-CoV-2 virus. J Clin Virol 129:104500. S1386-6532(20)30242-0. https://doi.org/10.1016/j. jcv.2020.104500

8. Scohy A, Anantharajah A, Bodeus M et al (2020) Low performance of rapid antigen detection test as frontline testing for COVID-19 diagnosis. J Clin Virol 129:104455. S13866532(20)30197-9. https://doi.org/10.1016/j.jcv.2020.104455

9. Salvagno GL, Gianfilippi G, Bragantini D et al (2021) Clinical assessment of the Roche SARS-CoV-2 rapid antigen test. Diagnosis (Berl). https://doi.org/10.1515/dx-2020-0154
10. Schildgen V, Demuth S, Lusebrink J, Schildgen O (2021) Limits and opportunities of SARS-CoV-2 antigen rapid tests: an experienced-based perspective. Pathogens 10(1):38. [pii] https://doi. org/10.3390/pathogens 10010038

11. Ciotti M, Maurici M, Pieri M et al (2021) Performance of a rapid antigen test in the diagnosis of SARS-CoV-2 infection. J Med Virol. https://doi.org/10.1002/jmv.26830

12. Lambert-Niclot S, Cuffel A, Le Pape S et al (2020) Evaluation of a rapid diagnostic assay for detection of SARS-CoV-2 antigen in nasopharyngeal swabs. J Clin Microbiol 58(8):e00977-20. https:// doi.org/10.1128/JCM.00977-20

13. Favresse J, Gillot C, Oliveira M et al (2021) Head-to-head comparison of rapid and automated antigen detection tests for the diagnosis of SARS-CoV-2 infection. J Clin Med 10(2):E265. https://doi.org/10.3390/jcm10020265

14. ECDC, WHO (2021) Methods for the detection and identification of SARS-CoV-2 variants. https://www.ecdc.europa.eu/sites/default/files/ documents/Methods-for-the-detection-and-identification-of-SARSCoV-2-variants-WHO-ECDC.pdf

15. Italian Ministry of Health (2021) 0000705-08/01/2021-DGPREDGPRE-P Aggiornamento della definizione di caso COVID19 e strategie di testing. https://www.fimmgag.org/2021/01/ 14/circolare-del-ministero-della-salute-0000705-08-01-2021dgpre-dgpre-p-aggiornamento-della-definizione-di-caso-covid19-e-strategie-di-testing/

Publisher's Note Springer Nature remains neutral with regard to jurisdictional claims in published maps and institutional affiliations. 